\title{
Anxiety and Obsession Following the COVID-19 Outbreak
}

\author{
Amir Adibi ${ }^{1}$, Hadis Jamshidbeigi ${ }^{2}$, Tayebe Jamshidbeigi ${ }^{3}$, Aliashraf Mozafari $^{2}$ and Ali Sahebi (iD ${ }^{2,}{ }^{*}$ \\ ${ }^{1}$ Department of Child and Adolescent Psychiatry, Ilam University of Medical Sciences, Ilam, Iran \\ ${ }^{2}$ Clinical Research Development Unit, Shahid Mostafa Khomeini Hospital, Ilam University of Medical Sciences, Ilam, Iran \\ ${ }^{3}$ Department of Internal Medicine, Ilam University of Medical Sciences, Ilam, Iran \\ "Corresponding author: Clinical Research Development Unit, Shahid Mostafa Khomeini Hospital, Ilam University of Medical Sciences, Ilam, Iran. Tel: +98-8433338228, Fax: \\ +98-8433338455, Email: Ali.sahebi.phd@gmail.com
}

Received 2020 May 31; Revised 2020 June 06; Accepted 2020 June 06.

Keywords: Anxiety, Coronavirus, Covid-19

\section{Dear Editor,}

Coronavirus disease 2019 (COVID-19) was first identified in Wuhan, China, and then spread rapidly around the world. The outbreak has led to concerns about public health around the world, especially in developing countries (1). COVID-19 has several psychiatric consequences (2). Adverse psychological outcomes such as post-traumatic stress disorder, confusion, and anger have been reported following the outbreak of diseases such as Ebola and severe acute respiratory syndrome (SARS), and delays in psychological intervention can lead to psychological trauma. Also, people with a history of psychiatric problems, including anxiety and depression, are more vulnerable to quarantine and social distance than others (3). The results of studies in Iran have shown that the rate of obsessivecompulsive disorder (OCD) and general anxiety disorders are $1 \%-11.9 \%$ and $0.54 \%-12.8 \%$, respectively, and anxiety disorders are the most common type of psychiatric disorders (4). Anxiety disorders, such as panic attacks and generalized anxiety disorder, may exacerbate physical symptoms, including shortness of breath (5). Following the outbreak of COVID-19, health and safety officials and caregivers, the media, social networks, and those around each individual frequently recommend personal hygiene. This issue increases the likelihood of hypersensitivity, extreme behavior, and subsequent OCD, and can exacerbate the disease in people with a history of anxiety and OCD. The fear of COVID-19 is natural and should be used to prevent the spread of the disease. If a person's fear of the disease is abnormal, it is a matter of concern and should be discussed with a psychotherapist. The authors of this article offer the following strategies for preventing obsessions:

People should follow valid health guidelines for the prevention of COVID-19 and note that the number of times and duration of handwashing should not exceed the recommended amount. People need to ask themselves whether the health behaviors they perform are for maintaining good health or getting rid of annoying and anxious thoughts. Furthermore, if it is to get rid of annoying and anxious thoughts, avoid them as much as possible. It is the best that people had been examined by psychiatrists or health care professionals and diagnosed OCD or anxiety disorder special anyone with a history of these problems.

\section{Footnotes}

Authors' Contribution: All authors contributed to the design and writing of this manuscript equally.

Conflict of Interests: The authors declare that there are no conflicts of interest.

Funding/Support: This paper has no sources of funding or other financial supports.

\section{References}

1. Moradi Y, Eshrati B. Estimation of the net reproductive number of COVID-19 in Iran. Med J Islam Republ Iran. 2020;34(1):240-6. doi: 10.34171/mjiri.34.34.

2. Saeedi M, Omrani-Nava V, Maleki I, Hedayatizadeh-Omran A, Ahmadi A, Moosazadeh M, et al. Opium addiction and COVID-19: Truth or false beliefs. Iran J Psychiatry Behav Sci. 2020;14(2). e103509. doi: 10.5812/ijpbs.103509.

3. Seyed Ahadi M, Sahraian MA, Rezaeimanesh N, Naser Moghadasi A. Psychiatric advice during COVID-19 pandemic for patients with multiple sclerosis. Iran J Psychiatry Behav Sci. 2020;14(2). e103243. doi: 10.5812/ijpbs.103243.

4. Heydari Yazdi AS, Eslamzadeh M, Mohammadi MR, Khaleghi A, Hooshyari Z, Moharreri F, et al. A survey of psychiatric disorders and their comorbidities in children and adolescents. Galen Med J. 2020;9. doi: 10.31661/gmj.v9io.1714.

5. Zarghami M. Psychiatric aspects of coronavirus (2019-nCoV) infection. Iran J Psychiatry Behav Sci. 2020;14(1). e102957. doi: 10.5812/ijpbs.102957. 